\title{
Ettude du système artériel du membre thoracique du Chameau
}

\author{
par. M.-A.-A. El HAGRI, F.R.C.V.S., B. SC. (Edin.) \\ Chef du Service d'anatomie \\ Faculté de Médecine vétérinaire, Giza, Égypte \\ et M.-B. MORCOS, B. V. SC., \\ du Service de Chirurgie
}

L'anatome du chameau, comparée à celle des autres animaux domestiques, n'a été étudiée que par un petit nombre de chercheurs dont la plupart étaient des Français. Chauveaú (1891) n'a donné que de brèves notes comparatives sur le squelette et le système musculaire du chameau. Bien que Lesbre (1903) ait décrit l'anatomie des différents appareils de cet animal, ses déscriptions sont, à bien des points de vue, brèves et incomplètes. Cauvet (1925) et Droandi (1939) ont cité cet auteur mot pour mot dans leurs publications et ont à peine apporté une contribution de quelque importance, au moins en ce qui concerne notre sujet. Leese (1927) a décrit de façon superficielle les caractéristiques générales de l'anatomie du chameau. De ce fait, durant ces dernières années, le chameau a constitué pour notre Service un excellent matériel d'étude pour des recherches approfondies sur l'anatomie des appareils digestif, uro-génital et respiratoire. A l'heure actuelle, nous effectuons une série de recherches sur les articulations des membres antérieurs et postérieurs du chameau, ce qui nous a permis d'étudier en même temps le système artériel de chacun de ces membres.

\section{MÉTHODE}

On a procédé à l'injection du système artériel du membre thoracique selon la technique décrite par Kellner (1934), 24 heures après une injection préalable d'une solution formolée à $10 \%$, glycérinée à $4 \%$ et phéniquée à $4 \%$.

\section{RÉSULTATS}

Lesbre (1903) a décrit brièvement le trajet du tronc artériel principal du membre thoracique et en a nommé les ramifications qui, dit-il, dans la région du bras, ressemblent à celles des autres animaux. Selon lui, parmi ces ramifications, l'artère collatérale interne du coude (cubitale) envoie des branches aux muscles perforé et perforant et l'artère interosseuse de l'avant-bras naft de l'artère radiale postérieure (radiale). Après avoir passé sous l'arcade radiocubitale, elle va se terminer dans les muscles extenseurs sur la face externe de l'avant-bras. Lesbre dit aussi que la radiale posterieure a pour branches terminales les artères radió-palmaire et palmaire métacarpienne. Le premier de ces vaisseaux descend à la surface du ligament postérieur du carpe et s'engage sous l'extrémité supérieure du ligament suspenseur du boulet où il s'unit avec la terminaison de l'artère cubitale en formant l'arcade sous-carpienne.

De cette arcade naissent trois artères interosseuses palmaires que Lesbre considère comme peu importantes. Il ajoute que l'artère palmaire métacarpienne prolonge le trajet de la radiale le long du bord interne du perforant, puis à la face postérieure des tendons fléchisseurs, pour se terminer enfin en deux artères digitales collatérales au-dessus de l'espace interdigité. Au-dessous du métacarpe elle donne une branche qui, après avoir traversé l'espace compris dans les angles de kifurcation des tendons fléchisseurs et du ligament suspenseur, reçoit la terminaison des artères interosseuses. Lesbre conclut que chaque artère digitale collatérale suit le côté concentrique du doigt correspondant et se termine dans les tissus sous-ongulés après avoir pénétré dans l'un des trous de la base de la troisième phalange. Chaque vaisseau envoie une ramification au coussinet plantaire et donne une branche comparable à l'artère perpendiculaire de Percival des Solipèdes.

Cauvet (1925) donne du système vasculaire une description semblable à celle de Lesbre (1903). Cependant il ajoute que l'une des trois artères interosseuses palmaires occupe une position inferne ef les deux autres une position externe et que sur le 

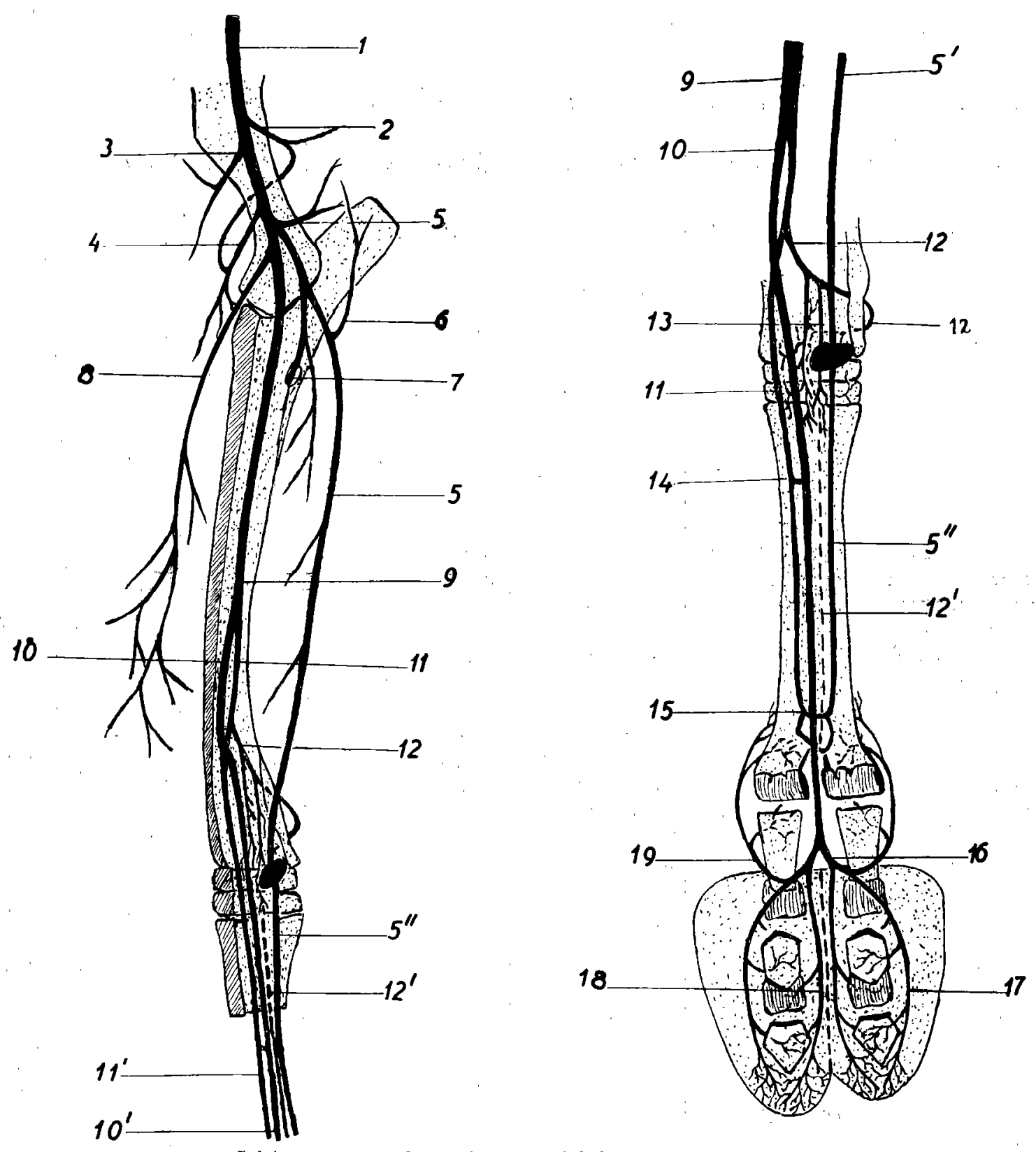

Schéma montrant le système artériel du membre antérieur.

1. - Humérale;

2. - Humérale profonde;

3. - A. du biceps;

4. - Radiale antérieure;

5. - Rameau articulaire du coude;

5' - Cubitale collatérale;

5" - Métacarpienne palmaire moyenne;

6. - Rameau articulaire;

7. - Interosseuse de l'avant-braz;

8. - Grosse artère cutané;

9. - Radiale postérieure;
10, 10' - Métacarpienne palmaire superficielle;

11, 11' - Métacarpienne palmaire profonde;

12. - A. interosseuse distale;

12' - Métacarpienne dorsale;

13. - Réseau carpien palmaire;

14. - Arcade sous-carpienne;

15. - Arcade palmaire profonde;

16. - Digitale palmaire;

17. - Rameau digital externe;

18. - Rameau digital interne:

19. - Rameau articulaire du boulet. 
bord externe des doigts il n'y a que des branches peu importantes. Droandi (1936) considère la radiale antérieure ou dorsale de l'avant-bras comme l'une des branches terminales de l'artère humérale. Il décrit aussi son trajet et dit qu'après avoir traversé la partie antérieure du coude elle accompagne le nerf radial puis perfore l'aponévrose en avant du radius et se termine par des ramifications qui s'unissent avec une branche de la radiale postérieure et, en partie, avec l'artère interosseuse de l'avant-bras.

Les résultats que nous avons obtenus au cours de nos recherches ne concordent pas avec les assertions des précédents auteurs. Nous avons observé notamment des différences sensibles dans le trajet et la distribution de certains vaisseaux.

La radiale antérieure ressemble à celle du cheval mais en diffère du fait qu'elle ne descend pas jusqu'à la région du carpe mais se termine dans les extenseurs de l'avant-bras comme c'est le cas chez le bœuf. L'anastomose entre ce vaisseau et l'artère humérale profonde se produit au-dessus de l'origine du muscle extenseur antérieur du métacarpe. La radiale antérieure fournit l'artère nourricière de l'humérus comme chez le bceuf et une branche articulaire qui, après avoir percé le tendon d'insertion du biceps, irrigue la partie antérieure du coude.

L'artère cubitale ou collatérale interne du coude de Lesbre se divise, tout près de son origine sur l'artère humérale, en une branche ascendante et une branche descendante.

La première se termine dans l'anconé et le chef intermédiaire du triceps brachial et dans la capsule articulaire du coude. La deuxième descend à la face interne du coude et est séparée de l'artère radiale postérieure par le nerf médian. En partie distale elle fournit les branches suivantes :

a) Une branche articulaire dı coude qui, après un trajet rétrograde sous l'origine du fléchisseur superficiel (perforé) et le chef huméral du fléchisseur profond des doigls (perforant), atleint la capsule articulaire.

b) L'artère interosseuse proximale qui irrigue le chef radial et le chef cubital du perforant et s'engage dans l'espace interosseux proximal de l'avant-bras.

Le vaisseau poursuit son trajet descendant dans l'avant-bras, caché à certains endroits par le chef huméral du perforant. Il traverse la gaine carpienne en compagnie du rameau profond du nerf cubital en position interne par rapport au pisiforme et se poursuit en formant l'artère métacarpienne palmaire moyenne, ainsi nommée pour des raisons que nous exposerons plus loin, le long des bords externes du fléchisseur superficiel des doigts et du ligament suspenseur dans le tiers proximal du métacarpe. Plus bas cette artère gagne progressivement la ligne médiane de la face palmaire du ligament suspenseur $\mathrm{du}$ boulet et rejoint finalement l'arcade palmaire profonde.

Immédiatement après la naissance de l'artère radiale antérieure, l'artère humérale donne un gros vaisseau cutané qui perce l'insertion aponévrotique du muscle pectoral transverse pour aller se terminer en plusieurs branches dans la peau de la face dorsale de l'avant-bras.

L'artère radiale postérieure présente un trajet semblable à celui qui a été décrit par d'autres auteurs. Cependant il peut être utile d'ajouter que dans le tiers proximal de l'avant-bras l'artère est située sur le bord interne du chef radial du perforant, en compagnie du nerf médian et de la veine satellite qui la côtoient, sans être couverte par le grand palmaire comme l'artère homologue du cheval; mais vers le milieu de l'avant-bras elle ressemble à cette dernière et à la branche cubitale de, l'artère radiale postérieure du bcuf, du fait qu'elle s'engage sous ce muscle. Dans le quart distal de l'avant-bras, l'artère radiale postérieure se termine en formant les artères radio-palmaire et palmaire métacarpienne telles qu'elles sont décrites par d'autres auteurs.

Cependant nous décrivons ici ces vaisseaux sous les noms respectifs d'artère métacarpienne palmaire profonde et métacarpienne palmaire superficielle pour des raisons que nous mentionnerons plus tard. La première de ces artères est la plus petite; elle fournit un vaisseau de bonne taille qui, après avoir donné les artères du réseau carpien palmaire, traverse l'orifice proximal de l'espace interosseux distal de l'avant-bras pour gagner la face dorsale du carpe où il fournit aussi des branches au réseau carpien dorsal. Pour des raisons évidentes on désignera dorénavant ce vaisseau sous le nom d'artère interosseuse distale. Du réseau carpien dorsal, un vaisseau semblable à l'artère métacarpienne dorsale du boeuf descend dans la gouttière vasculaire dorsale de l'os métaçarpien et atteint l'arcade palmaire profonde en passant par l'espace interdigite. L'artère métacarpienne palmaire profonde descend ensuite à la face interne du ligament latéral interne du carpe. Dans le tiers proximal du métacarpe, elle s'unit à l'artère métacarpienne palmaire superficielle pour former l'arcade sous-carpienne en position profonde par rapport au bord interne du ligament suspenseur. De cette arcade, l'artère poursuit son trajet le-long du métacarpe dans la:faible rainure vasculaire palmaire et rejoint l'arcade palmaire profonde. Elle fournit les artères nourricières des métacarpiens III et IV.

Par ailleurs, l'artère métacarpienne palmaire superficielle descend dans la gaine carpienne avec le nerf médian. Elle suit le bord interne des tendons fléchisseurs et après avoir concouru à la formation de 
l'arcade sous-carpienne, comme on l'a déjà dit, elle occupe une position plus centrale sur la face palmaire des tendons fléchisseurs. Dans l'échancrure interdigitale elle fournit une branche qui se termine dans l'arcade palmaire profonde. Celle-ci est ainsi formée par l'union des artères métacarpiennes palmaires (profonde, moyenne et superficielle) et de l'artère métacarpienne dorsale.

De cette arcade part de chaque côté une branche qui passe transversalement entre le métacarpe et le ligament suspenseur du boulet pour se ramifier dans la région des'talons. En partie distale par rapport à l'arcade palmaire profonde, l'artère métacarpienne palmaire superficielle se divise au milieu de la première phalange en deux artères dígitales palmaires. Chacun de ces vaisseaux, après un très court trajet; se divise à son tour en un rameau externe et un rameau interne qui suivent les bords correspondants des doigts et se terminent par de fines branches dans le tissu sous-ongulé, à l'apex de la troisième phâlange. En outre, le rameau externe de chacune des artères digitales palmaires fournit une branche à l'articulation du boulet, branche qui suit un trajet rétrograde le long du bord externe de la premiere phalange. Les branches terminales des artères digitales palmaires fournissent aussi, au cours de leur trajet, des rameaux dorsaux ef palmaires à chacune des phalanges ; parmi ces rameaux ceux de la première phalange correspondent à l'artère perpendiculaire de Percival des Solipèdes.

\section{DISCUSSION}

La description donnée par Droandi (1939) du trajet de la radiale antérieure semble s'appliquer davantage à celui de la grosse branche cutanée de l'artc̀re humćralo plutôt qu'à celui de la radiale antérieure, ce qui n'a été signalé par aucun des précédents auteurs. Céci est aussi étayé par le fait que l'artère radiale antérieure ne descend pas jusqu'à la région du carpe mais se termine dans les extenseurs de l'avant-bras comme chez le bœuf. Selon les précédents auteurs, I'artère interosseuse de l'avant-bras prend naissance sur la radiale postérieurc ct se tormine dans les extenseur's sur la face externe de l'avant-bras après avoir traversé l'arcade radio-cubitale. Bien qu'il soit fréquent d'observer des variations dans l'origine des vaisseaux sanguins, comme c'est le cas pour celle de l'artère interosseuse à partir de l'artère cubitale au lieu de la radiale postérieure, comment cependant ce vaisseau pourrait-il traverser l'arcade radio-cubitale alors que la fusion précoce et complète des os de l'avant-bras chez le chameau ne lui laisse aucune issue vers l'exterieur? Pour la même raison, il est difficile d'admettre l'asser- non de Droandi (1936) sur l'existence d'une anastomose entre les artères interosseuse et radiale antérieure.

Aucun des auteurs précédents n'a signalé la présence de l'artère interosseuse distale chez le chameau. Nous avons constaté que ce vaisseau est une branche de l'artère métacarpienne palmaire profonde (radio-palmaire) dont le trajet et le prolongement, cunstilué par l'artère métacarpienne dorsale, ont été ci-dessus complètement décrits. Contrairement aux assertions de Lesbre (1903) et Cauvet (1925) sur' le mode de formation de l'arcade souscarpienne, nous avons constaté que l'artère' cubitale effectue directement son trajet jusqu'à l'arcade palmaire profonde et, de ce fait, ne joue aucun rôle dans la formation de l'arcade sous-carpienne.

Les prolongements des artères cubitale, radiopalmaire et palmaire métacarpienne, dans la région métacarpienne, ont été décrits ci-dessus sous les noms respectifs d'arteres métacarpiennes palmaires moyenne, profonde et superficielle, en raison de lcurs positions topographiques respectives dans la partie inférieure du membre.

L'assertion de Cauvet (1925) selon laquelle les artères digitales collatérales se trouvent seulement sur les bords internes des doigts tandis que les bords externes ne possèdent quie des branches peu importantes, résulte probablement d'une méconnaissance de la distribution complète de ces vaisseaux. Une dissection soigneuse a montré clairement que chacun des doigts est également irrigué de chaque côté par une branche correspondante de l'artère digitale collatérale. De même, on a constaté que l'artère du. coussinet plantaire part, de chaque côté, de l'arcade palmaire profonde et non de l'artère digitale collatérale comme l'ont dit les auteurs précédents.

\section{RÉSUMEE}

Nous décrivons le trajet complet de l'artère cubitale (collatérale interne du coude) et affirmons que, contrairement aux assertions d'autres chercheurs, elle ne joue aucun rôle dans la formation de l'arcade sous-carpienne.

Pour des raisons anatomiques que nous exposons ci-dessus, nous affirmons que le trajet de l'artère jnterosseuse de l'avant-bras et son union avec un autre vaisseau ne peuvent matériellement pas être tels que les ont décrits d'autres auteurs.

En fait les artères interosseuses palmaires signalées dans la littérature sont les prolongements de la cubitale collatérale, do la radio-palmaire et de la palmaire metacarpienne des autres auteurs. La position topographique de chacun de ces vaisseaux dans 
la région de la main permet d'établir une nouvelle nomenclature fondée sur leurs positions réelles.

Nous donnons aussi une description complète de la vascularisation de la région du pied.

Nous avons constaté qu'il existe chez le chameau une artère interosseuse distale qui se prolonge par une artère métacarpienne dorsale, ce qui n'avait jamars ele mertionne jusqu'icl

Le mode de formation de l'arcade sous-carpienne et de l'arcade melacar pienne palmarre prcfonde est aussi étudie.

\section{BIBLIOGRAPHIE}

CAUVE'T, 1925. - Le Chameau

CHAUVEAU (A.), 1891 - The comparative anatomy of the domesticated animals

DROANDI (I.), 1936. - Il Camello

KELLNER (C.). 1934. - Anai. Rec. 59, 393.

LEESE (A.-S.), 1927. - A treatise on the one humped Camel.

LESBRE (M.-F.X.), 1903. - Recherches anatomiques sur les camélidés. 